\title{
ON QUASISIMILARITY FOR LOG-HYPONORMAL OPERATORS
}

\author{
I. H. JEON ${ }^{1}$ \\ Department of Mathematics, Ewha Women's University, Seoul 120-750, Korea \\ e-mail: jihmath@ewha.ac.kr \\ K. TANAHASHI ${ }^{2}$ \\ Department of Mathematics, Tohoku Pharmaceutical University, Sendai 981-8558, Japan \\ e-mail: tanahasi@tohoku-pharm.ac.jp \\ and A. UCHIYAMA \\ Sendai National College of Technology, Sendai 989-3128, Japan \\ e-mail: uchiyama@cc.sendai-ct.ac.jp
}

(Received 11 November, 2002; accepted 23 June, 2003)

\begin{abstract}
In this paper we show that the normal parts of quasisimilar loghyponormal operators are unitarily equivalent. A Fuglede-Putnam type theorem for log-hyponormal operators is proved. Also, it is shown that a log-hyponormal operator that is quasisimilar to an isometry is unitary and that a log-hyponormal spectral operator is normal.
\end{abstract}

2000 Mathematics Subject Classification. 47B20.

1. Introduction. Let $\mathcal{H}$ and $\mathcal{K}$ be infinite dimensional complex Hilbert spaces and let $L(\mathcal{H}, \mathcal{K})$ denote the set of bounded linear operators from $\mathcal{H}$ to $\mathcal{K}$. If $\mathcal{H}=\mathcal{K}$, we write $L(\mathcal{H})$ in place of $L(\mathcal{H}, \mathcal{K})$. Let $T \in L(\mathcal{H})$. $T$ is said to be p-hyponormal if $\left(T^{*} T\right)^{p}-\left(T T^{*}\right)^{p} \geq 0$ for some $0<p \leq 1$. It is known that if $T$ is $p$-hyponormal and $0<q<p$, then $T$ is $q$-hyponormal, by the Lowner-Heinz's inequality [11, 14]. If $p=1$, $T$ is said to be hyponormal and if $p=1 / 2, T$ is said to be semi-hyponormal. $T$ is said to be log-hyponormal if $T$ is invertible and satisfies the following inequality

$$
\log \left(T^{*} T\right) \geq \log \left(T T^{*}\right) .
$$

It is known that invertible $p$-hyponormal operators are log-hyponormal but that the converse is not true [16]. However it is very interesting that we may regard log-hyponormal operators as 0-hyponormal operators [16, 17]. Let $T=U|T|$ be the polar decomposition of $T$. We usually define the Aluthge transform of $T$ by $\widetilde{T}=|T|^{1 / 2} U|T|^{1 / 2}$. Let $\widetilde{T}=V|\widetilde{T}|$ be the polar decomposition of $\widetilde{T}$, and define the second Aluthge transform of $T$ by $\widehat{T}=|\widetilde{T}|^{1 / 2} V|\widetilde{T}|^{1 / 2}$. It is known that if $T$ is loghyponormal, then $\widetilde{T}$ is semi-hyponormal and $\widehat{T}$ is hyponormal $[\mathbf{1}, \mathbf{1 6}, \mathbf{2 1}]$. An operator $X \in L\left(\mathcal{H}_{2}, \mathcal{H}_{1}\right)$ is called a quasiaffinity if $X$ is injective and has dense range $R(X)$. For $T_{1} \in L\left(\mathcal{H}_{1}\right)$ and $T_{2} \in L\left(\mathcal{H}_{2}\right)$, if there exist quasiaffinities $X \in L\left(\mathcal{H}_{2}, \mathcal{H}_{1}\right)$ and

\footnotetext{
${ }^{1}$ This work was supported by Korea Research Foundation Grant (KRF-2001-050-D0001).

${ }^{2}$ This research was supported by Grant-in-Aid Research No. 10640187.
} 
$Y \in L\left(\mathcal{H}_{1}, \mathcal{H}_{2}\right)$ such that $T_{1} X=X T_{2}$ and $Y T_{1}=T_{2} Y$, then we say that $T_{1}$ and $T_{2}$ are quasisimilar.

Xia [20] investigated properties of hyponormal and semi-hyponormal operators. Aluthge [1] introduced $p$-hyponormal operators and investigated their properties using Aluthge transforms. The idea of a log-hyponormal operator is due to Ando [3] and the first paper in which log-hyponormality appeared is [9]. See $[\mathbf{2}, \mathbf{1 6}, \mathbf{1 7}, \mathbf{1 8}]$ for properties of log-hyponormal operators.

Jeon and Duggal [13] proved that the normal parts of quasisimilar $p$-hyponormal operators are unitarily equivalent, a $p$-hyponormal operator compactly quasisimilar to an isometry is unitary, and a $p$-hyponormal spectral operator is normal.

In this paper we prove that similar results hold for log-hyponormal operators; i.e., the normal parts of quasisimilar log-hyponormal operators are unitarily equivalent, Fuglede-Putnam's theorem holds for log-hyponormal operators, a log-hyponormal operator quasisimilar to an isometry is normal, and a log-hyponormal spectral operator is normal.

\section{Normal parts of quasisimilar log-hyponormal operators.}

THEOREM 1. Let $\mathcal{M}$ be an invariant subspace of a log-hyponormal operator $T \in L(\mathcal{H})$ and $\left.T\right|_{\mathcal{M}}$ the restriction of $T$ to $\mathcal{M}$. If $\left.T\right|_{\mathcal{M}}$ is invertible, then $\left.T\right|_{\mathcal{M}}$ is log-hyponormal.

Proof. Put $T=\left(\begin{array}{ll}A & B \\ 0 & C\end{array}\right)$ and $P=\left(\begin{array}{ll}1 & 0 \\ 0 & 0\end{array}\right)$ on $\mathcal{H}=\mathcal{M} \oplus \mathcal{M}^{\perp}$. Since

$$
\log X=\lim _{p \downarrow 0} \frac{X^{p}-1}{p}
$$

for an arbitrary positive invertible operator $X$, we have

$$
\begin{aligned}
P\left(\log T^{*} T\right) P & =\lim _{p \downarrow 0}\left(\begin{array}{ll}
1 & 0 \\
0 & 0
\end{array}\right) \frac{\left(T^{*} T\right)^{p}-1}{p}\left(\begin{array}{ll}
1 & 0 \\
0 & 0
\end{array}\right) \\
& \leq \lim _{p \downarrow 0} \frac{1}{p}\left\{\left(\left(\begin{array}{ll}
1 & 0 \\
0 & 0
\end{array}\right) T^{*} T\left(\begin{array}{ll}
1 & 0 \\
0 & 0
\end{array}\right)\right)^{p}-\left(\begin{array}{ll}
1 & 0 \\
0 & 0
\end{array}\right)\right\} \\
& =\lim _{p \downarrow 0}\left(\begin{array}{cc}
\frac{\left(A^{*} A\right)^{p}-1}{p} & 0 \\
0 & 0
\end{array}\right)=\left(\begin{array}{cc}
\log A^{*} A & 0 \\
0 & 0
\end{array}\right),
\end{aligned}
$$

by Hansen's inequality [10], and

$$
\begin{aligned}
P\left(\log T T^{*}\right) P & =\lim _{p \downarrow 0}\left(\begin{array}{ll}
1 & 0 \\
0 & 0
\end{array}\right) \frac{\left(T T^{*}\right)^{p}-1}{p}\left(\begin{array}{ll}
1 & 0 \\
0 & 0
\end{array}\right) \\
& \geq \lim _{p \downarrow 0}\left(\begin{array}{ll}
1 & 0 \\
0 & 0
\end{array}\right) \frac{\left(T\left(\begin{array}{ll}
1 & 0 \\
0 & 0
\end{array}\right) T^{*}\right)^{p}-1}{p}\left(\begin{array}{ll}
1 & 0 \\
0 & 0
\end{array}\right) \\
& =\lim _{p \downarrow 0}\left(\begin{array}{cc}
\frac{\left(A A^{*}\right)^{p}-1}{p} & 0 \\
0 & 0
\end{array}\right)=\left(\begin{array}{cc}
\log A A^{*} & 0 \\
0 & 0
\end{array}\right),
\end{aligned}
$$


by Löwner-Heinz's inequality $[\mathbf{1 1}, \mathbf{1 4}]$. Since $T$ is log-hyponormal,

$$
\begin{aligned}
\left(\begin{array}{cc}
\log A A^{*} & 0 \\
0 & 0
\end{array}\right) & \leq P\left(\log T T^{*}\right) P \\
& \leq P\left(\log T^{*} T\right) P \leq\left(\begin{array}{cc}
\log A^{*} A & 0 \\
0 & 0
\end{array}\right)
\end{aligned}
$$

Hence, $A=\left.T\right|_{\mathcal{M}}$ is also log-hyponormal.

TheOREM 2. Let $T \in L(\mathcal{H})$ be log-hyponormal. Then $T=T_{1} \oplus T_{2}$ on the space $\mathcal{H}=\mathcal{H}_{1} \oplus \mathcal{H}_{2}$, where $T_{1}$ is normal and $T_{2}$ is pure and log-hyponormal; i.e., $T_{2}$ has no invariant subspace $\mathcal{M}$ such that $\left.T_{2}\right|_{\mathcal{M}}$ is normal.

Proof. It is easy to prove that $T=T_{1} \oplus T_{2}$ on $\mathcal{H}=\mathcal{H}_{1} \oplus \mathcal{H}_{2}$, where $T_{1}$ is normal and $T_{2}$ is pure, by [18, Lemma 12]. Since $\sigma\left(T_{2}\right) \subset \sigma(T), T_{2}$ is invertible and loghyponormal, by Theorem 1 .

The next lemma was proved for dominant operators in [15, Theorem 1] and for $p$ hyponormal operators in [13]. Recall that an operator $T \in B(\mathcal{H})$ is said to be dominant if for any $\lambda \in \mathbb{C}$ there exists an $M_{\lambda} \geq 0$ such that $\left\|(T-\lambda)^{*} x\right\| \leq M_{\lambda}\|(T-\lambda) x\|$, for all $x \in \mathcal{H}$. If $M_{\lambda}$ is a constant, then $T$ is said to be $M$-hyponormal.

Lemma 3. Let $T_{1} \in L\left(\mathcal{H}_{1}\right)$ be a log-hyponormal operator and let $T_{2} \in L\left(\mathcal{H}_{2}\right)$ be a normal operator. If there exists an operator $X \in L\left(\mathcal{H}_{2}, \mathcal{H}_{1}\right)$ with dense range such that $T_{1} X=X T_{2}$, then $T_{1}$ is normal.

Proof. First, we decompose $T_{1}$ into its normal and pure parts by $T_{1}=T_{11} \oplus T_{12}$ with respect to a decomposition $\mathcal{H}_{1}=\mathcal{H}_{11} \oplus \mathcal{H}_{12}$. Let $T_{12}=U_{12}\left|T_{12}\right|$ be the polar decomposition of $T_{12}$ and $\widetilde{T}_{12}=\left|T_{12}\right|^{1 / 2} U_{12}\left|T_{12}\right|^{1 / 2}$. Let $\widetilde{T}_{12}=V_{12}\left|\widetilde{T}_{12}\right|$ be the polar decomposition of $\widetilde{T}_{12}$ and $\widehat{T}_{12}=\left|\widetilde{T}_{12}\right|^{1 / 2} V_{12}\left|\widetilde{T}_{12}\right|^{1 / 2}$. Since $T_{11}$ is normal, we have that $\widetilde{T}_{1}=T_{11} \oplus \widetilde{T}_{12}$ and $\widehat{T}_{1}=T_{11} \oplus \widehat{T}_{12}$. Let $W=\left|\widetilde{T}_{12}\right|^{1 / 2}\left|T_{12}\right|^{1 / 2}$. Since $N\left(\left|T_{12}\right|\right)=$ $N\left(T_{12}\right)=\{0\}$, by Theorem $2,\left|T_{12}\right|^{\frac{1}{2}}$ is a quasiaffinity. Hence $\widehat{T}_{12}$ is injective and $W$ is a quasiaffinity such that $\widehat{T}_{12} W=W T_{12}$. Let $Y=I_{H_{11}} \oplus W$. Then $\widehat{T}_{1}$ is hyponormal and $Y$ is a quasiaffinity such that $\widehat{T}_{1} Y=Y T_{1}$. Thus we have that $\widehat{T}_{1}(Y X)=(Y X) T_{2}$ and $Y X$ has dense range. Hence $\widehat{T}_{1}$ is normal, by [15, Theorem 1], and so $T_{1}$ is normal by [16, Theorem 7].

The following lemma is due to Williams [19, Lemma 1.1].

LemMa 4. [19] Let $N_{i} \in L\left(\mathcal{H}_{i}\right)$ be normal for each $i=1$, 2. If $X \in L\left(\mathcal{H}_{2}, \mathcal{H}_{1}\right)$ and $Y \in L\left(\mathcal{H}_{1}, \mathcal{H}_{2}\right)$ are injective such that $N_{1} X=X N_{2}$ and $Y N_{1}=N_{2} Y$, then $N_{1}$ and $N_{2}$ are unitarily equivalent.

Conway [4] proved that the normal parts of quasisimilar subnormal operators are unitarily equivalent and gave an example showing that the pure parts of quasisimilar subnormal operators need not be quasisimilar. This result was generalized to classes of dominant operators in [15] and $p$-hyponormal operators in [13], respectively. We prove that these results hold for log-hyponormal operators.

THEOREM 5. For each $i=1,2$, let $T_{i} \in L\left(\mathcal{H}_{i}\right)$ be log-hyponormal operators and let $T_{i}=N_{i} \oplus V_{i}$ on $\mathcal{H}_{i}=\mathcal{H}_{i 1} \oplus \mathcal{H}_{i 2}$, where $N_{i}$ and $V_{i}$ are the normal and pure parts, respectively, of $T_{i}$. If $T_{1}$ and $T_{2}$ are quasisimilar, then $N_{1}$ and $N_{2}$ are unitarily equivalent 
and there exist $X_{*} \in L\left(\mathcal{H}_{22}, \mathcal{H}_{12}\right)$ and $Y_{*} \in L\left(\mathcal{H}_{12}, \mathcal{H}_{22}\right)$ having dense ranges such that $V_{1} X_{*}=X_{*} V_{2}$ and $Y_{*} V_{1}=V_{2} Y_{*}$.

Proof. By hypothesis there exist quasiaffinities $X \in L\left(\mathcal{H}_{2}, \mathcal{H}_{1}\right)$ and $Y \in L\left(\mathcal{H}_{1}, \mathcal{H}_{2}\right)$ such that $T_{1} X=X T_{2}$ and $Y T_{1}=T_{2} Y$. Let

$$
X=\left(\begin{array}{ll}
X_{1} & X_{2} \\
X_{3} & X_{4}
\end{array}\right) \text { and } Y=\left(\begin{array}{ll}
Y_{1} & Y_{2} \\
Y_{3} & Y_{4}
\end{array}\right)
$$

with respect to $\mathcal{H}_{2}=\mathcal{H}_{21} \oplus \mathcal{H}_{22}$ and $\mathcal{H}_{1}=\mathcal{H}_{11} \oplus \mathcal{H}_{12}$, respectively. A simple matrix calculation shows that

$$
V_{1} X_{3}=X_{3} N_{2} \text { and } V_{2} Y_{3}=Y_{3} N_{1} \text {. }
$$

We claim that $X_{3}=Y_{3}=0$. Let $\mathcal{M}=\overline{R\left(X_{3}\right)}$. Then $\mathcal{M}$ is a non-trivial invariant subspace of $V_{1}$. Since $V_{1}^{*} X_{3}=X_{3} N_{2}^{*}$, by [18, Theorem 3], $\mathcal{M}$ is an invariant subspace of $V_{1}^{*}$. Hence $\mathcal{M}$ reduces $V_{1}, \sigma\left(\left.V_{1}\right|_{\mathcal{M}}\right) \subset \sigma\left(V_{1}\right)$ and $\left.V_{1}\right|_{\mathcal{M}}$ is invertible. Let $V_{1}^{\prime}=\left.V_{1}\right|_{\mathcal{M}}$ and define an operator $X_{3}^{\prime}: \mathcal{H}_{21} \rightarrow \mathcal{M}$ by $X_{3}^{\prime} x=X_{3} x$, for each $x \in \mathcal{H}_{21}$. Then $V_{1}^{\prime}$ is loghyponormal, by Theorem 1, so that $X_{3}^{\prime}$ has dense range and satisfies $V_{1}^{\prime} X_{3}^{\prime}=X_{3}^{\prime} N_{2}$. Hence $V_{1}^{\prime}$ is normal, by Lemma 3 . Since $V_{1}$ is pure, this implies that $\mathcal{M}=\{0\}$ and $X_{3}=0$. Similarly, we have $Y_{3}=0$. Hence $X_{1}$ and $Y_{1}$ are injective.

Since $N_{1} X_{1}=X_{1} N_{2}$ and $Y_{1} N_{1}=N_{2} Y_{1}, N_{1}$ and $N_{2}$ are unitarily equivalent, by Lemma 4. Also, $X_{4}$ and $Y_{4}$ have dense ranges. Hence $V_{1} X_{4}=X_{4} V_{2}$ and $Y_{4} V_{1}=V_{2} Y_{4}$, so that the proof is complete.

Corollary 6. Let $T_{1} \in L\left(\mathcal{H}_{1}\right)$ and $T_{2} \in L\left(\mathcal{H}_{2}\right)$ be quasisimilar log-hyponormal operators. If $T_{1}$ is pure, then $T_{2}$ is also pure.

COROLlaRY 7. Let $T_{1} \in L\left(\mathcal{H}_{1}\right)$ be log-hyponormal and let $T_{2} \in L\left(\mathcal{H}_{2}\right)$ be normal. If $T_{1}$ and $T_{2}$ are quasisimilar, then $T_{1}$ and $T_{2}$ are unitarily equivalent normal operators.

\section{A Fuglede-Putnam type theorem for log-hyponormal operators.}

TheOREM 8. Let $T_{1} \in L\left(\mathcal{H}_{1}\right)$ and $T_{2}^{*} \in L\left(\mathcal{H}_{2}\right)$ be log-hyponormal or p-hyponormal operators satisfying $T_{1} X=X T_{2}$, for some operator $X \in L\left(\mathcal{H}_{2}, \mathcal{H}_{1}\right)$. Then $T_{1}^{*} X=$ $X T_{2}^{*}, \overline{R(X)}$ reduces $T_{1}, N(T)^{\perp}$ reduces $T_{2}$, and $\left.T_{1}\right|_{\overline{R(X)}},\left.T_{2}\right|_{N(X)^{\perp}}$ are unitarily equivalent normal operators.

Proof. Duggal [5, Theorem7] proved the case in which $T_{1}$ and $T_{2}^{*}$ are $p$-hyponormal. First we prove the case in which $T_{1}$ and $T_{2}^{*}$ are log-hyponormal. Let $T_{1}=U_{1}\left|T_{1}\right|$ and $T_{2}^{*}=U_{2}^{*}\left|T_{2}^{*}\right|$ be the polar decompositions of $T_{1}$ and $T_{2}^{*}$, respectively. Let $\widetilde{T}_{1}=$ $\left|T_{1}\right|^{1 / 2} U_{1}\left|T_{1}\right|^{1 / 2}, \widetilde{T_{2}^{*}}=\left|T_{2}^{*}\right|^{1 / 2} U_{2}^{*}\left|T_{2}^{*}\right|^{1 / 2}$ and $W=\left|T_{1}\right|^{\frac{1}{2}} X\left|T_{2}^{*}\right|^{\frac{1}{2}}$. Since $T_{1} X=X T_{2}$, we have $\widetilde{T}_{1} W=W\left(\widetilde{T_{2}^{*}}\right)^{*}$. Since $\widetilde{T}_{1}$ and $\widetilde{T}_{2}^{*}$ are semi-hyponormal, by [16], $\overline{R(W)}$ reduces $\widetilde{T}_{1}$ and $N(W)^{\perp}$ reduces $\left(\widetilde{T}_{2}^{*}\right)^{*}$. Also $\left.\widetilde{T}_{1}\right|_{R(W)}$ and $\left.\left(\widetilde{T}_{2}^{*}\right)^{*}\right|_{N(W)^{\perp}}$ are unitarily equivalent normal operators, by [5, Theorem 7]. By [18, Lemma 3], $T_{1}$ and $T_{2}^{*}$ are of the forms

$$
\begin{aligned}
& T_{1}=\left.\widetilde{T}_{1}\right|_{\overline{R(W)}} \oplus S_{1}=N_{1} \oplus S_{1} \text { on } \overline{R(W)} \oplus R(W)^{\perp}, \\
& T_{2}^{*}=\left.\widetilde{T}_{2}^{*}\right|_{N(W)^{\perp}} \oplus S_{2}^{*}=N_{2}^{*} \oplus S_{2}^{*} \text { on } N(W)^{\perp} \oplus N(W),
\end{aligned}
$$


where $N_{1}$ and $N_{2}$ are unitarily equivalent normal operators. Since $T_{1}$ and $T_{2}^{*}$ are invertible, $N_{1}, N_{2}, S_{1}$ and $S_{2}$ are also invertible. Let

$$
X=\left(\begin{array}{ll}
X_{11} & X_{12} \\
X_{21} & X_{22}
\end{array}\right) \text { and } W=\left(\begin{array}{cc}
W_{11} & 0 \\
0 & 0
\end{array}\right)
$$

with respect to $\mathcal{H}_{1}=\overline{R(W)} \oplus R(W)^{\perp}$ and $\mathcal{H}_{2}=N(W)^{\perp} \oplus N(W)$, respectively. Then $W=\left|T_{1}\right|^{\frac{1}{2}} X\left|T_{2}^{*}\right|^{\frac{1}{2}}$ implies that

$$
\left(\begin{array}{cc}
W_{11} & 0 \\
0 & 0
\end{array}\right)=\left(\begin{array}{ll}
\left|N_{1}\right|^{\frac{1}{2}} X_{11}\left|N_{2}^{*}\right|^{\frac{1}{2}} & \left|N_{1}\right|^{\frac{1}{2}} X_{12}\left|S_{2}^{*}\right|^{\frac{1}{2}} \\
\left|S_{1}\right|^{\frac{1}{2}} X_{21}\left|N_{2}^{*}\right|^{\frac{1}{2}} & \left|S_{1}\right|^{\frac{1}{2}} X_{22}\left|S_{2}^{*}\right|^{\frac{1}{2}}
\end{array}\right)
$$

Hence $X_{12}=0, X_{21}=0, X_{22}=0$ and $X_{11}: N(W)^{\perp} \rightarrow \overline{R(X)}$ is a quasiaffinity. This implies that $\overline{R(X)}=\overline{R\left(X_{11}\right)}=\overline{R(W)}, N(X)=N(W)$ and $\left.T_{1}\right|_{\overline{R(X)}},\left.T_{2}\right|_{N(X)^{\perp}}$ are unitarily equivalent normal operators. Since $T_{1} X=X T_{2}$, we have that $N_{1} X_{11}=X_{11} N_{1}$. Thus $N_{1}^{*} X_{11}=X_{11} N_{1}^{*}$ and $T_{1}^{*} X=X T_{2}^{*}$.

Next we prove the case in which $T_{1}$ is $p$-hyponormal and $T_{2}^{*}$ is log-hyponormal. In this case we have the equation (3.1), where $N_{2}$ and $S_{2}$ are invertible by the argument of the case above. Since $N_{1}$ and $N_{2}$ are unitarily equivalent, $N_{1}$ is invertible. It follows that $X_{12}=0,\left|S_{1}\right|^{\frac{1}{2}} X_{21}=0,\left|S_{1}\right|^{\frac{1}{2}} X_{22}=0$ and $S_{1} X_{21}=0, S_{1} X_{22}=0$. Then $T_{1} X=X T_{2}$ implies that

$$
\left(\begin{array}{cc}
N_{1} X_{11} & 0 \\
0 & 0
\end{array}\right)=\left(\begin{array}{cc}
X_{11} N_{2} & 0 \\
X_{21} N_{2} & X_{22} S_{2}
\end{array}\right)
$$

Hence $X_{21}=0, X_{22}=0$ and $X_{11}$ is a quasiaffinity. The rest of the proof is similar to the case above.

Lastly we prove the case in which $T_{1}$ is log-hyponormal and $T_{2}^{*}$ is $p$-hyponormal. Here we have the equation (3.1), where $N_{1}$ and $S_{1}$ are invertible. Since $N_{1}$ and $N_{2}$ are unitarily equivalent, $N_{2}$ is invertible. Hence $X_{21}=0, X_{12}\left|S_{2}^{*}\right|^{\frac{1}{2}}=0, X_{22}\left|S_{2}^{*}\right|^{\frac{1}{2}}=0$ and $X_{12} S_{2}=0, X_{22} S_{2}=0$. Then $T_{1} X=X T_{2}$ implies that

$$
\left(\begin{array}{cc}
N_{1} X_{11} & N_{1} X_{12} \\
0 & S_{1} X_{22}
\end{array}\right)=\left(\begin{array}{cc}
X_{11} N_{2} & 0 \\
0 & 0
\end{array}\right)
$$

Hence $X_{12}=0, X_{22}=0$ and $X_{11}$ is a quasiaffinity. The rest of the proof is similar to the case above.

REMARK. Let $T=U|T|$ be the polar decomposition of $T$. We define $\widetilde{T}=$ $|T|^{\frac{1}{2}} U|T|^{\frac{1}{2}}$. Since $T^{*}=|T| U^{*}=U^{*} U|T| U^{*}=U^{*}\left|T^{*}\right|, T^{*}$ has the polar decomposition $T^{*}=U^{*}\left|T^{*}\right|$. Hence $\widetilde{T^{*}}=\left|T^{*}\right|^{\frac{1}{2}} U^{*}\left|T^{*}\right|^{\frac{1}{2}}=U(\widetilde{T})^{*} U^{*}$ and $(\widetilde{T})^{*}=U^{*} \widetilde{T^{*}} U$. Thus $\widetilde{T}^{*}$ is log-hyponormal (resp., $p$-hyponormal) if and only if $(\widetilde{T})^{*}$ is log-hyponormal (resp, $p$-hyponormal).

A generalization to dominant operator is given by the following Corollary.

Corollary 9. Let $T_{1} \in L\left(\mathcal{H}_{1}\right)$ be dominant and $T_{2}^{*} \in L\left(\mathcal{H}_{2}\right)$ log-hyponormal. If $T_{1} X=X T_{2}$, for some operator $X \in L\left(\mathcal{H}_{2}, \mathcal{H}_{1}\right)$, then $T_{1}^{*} X=X T_{2}^{*}, \overline{R(X)}$ reduces $T_{1}, N(T)^{\perp}$ reduces $T_{2}$, and $\left.T_{1}\right|_{\overline{R(X)}},\left.T_{2}\right|_{N(X)^{\perp}}$ are unitarily equivalent normal operators. 
Proof. Decompose $T_{1}$ and $T_{2}^{*}$ into their normal and pure parts. Then we have

$$
\begin{aligned}
& T_{1}=N_{1} \oplus S_{1} \text { on } \mathcal{H}_{1}=\mathcal{H}_{11} \oplus \mathcal{H}_{12}, \\
& T_{2}=N_{2} \oplus S_{2} \text { on } \mathcal{H}_{2}=\mathcal{H}_{21} \oplus \mathcal{H}_{22},
\end{aligned}
$$

and

$$
X=\left(\begin{array}{ll}
X_{11} & X_{12} \\
X_{21} & X_{22}
\end{array}\right): \mathcal{H}_{2}=\mathcal{H}_{21} \oplus \mathcal{H}_{22} \rightarrow \mathcal{H}_{1}=\mathcal{H}_{11} \oplus \mathcal{H}_{12},
$$

where $N_{1}, N_{2}$ are normal, $S_{1}$ is dominant and $S_{2}^{*}$ is log-hyponormal. Then $T_{1} X=X T_{2}$ implies that

$$
\left(\begin{array}{ll}
N_{1} X_{11} & N_{1} X_{12} \\
S_{1} X_{21} & S_{1} X_{22}
\end{array}\right)=\left(\begin{array}{ll}
X_{11} N_{2} & X_{12} S_{2} \\
X_{21} N_{2} & X_{22} S_{2}
\end{array}\right)
$$

Let $S_{2}^{*}=U_{2}^{*}\left|S_{2}^{*}\right|$ be the polar decomposition of $S_{2}^{*}$ and $\widetilde{S}_{2}^{*}=\left|S_{2}^{*}\right|^{\frac{1}{2}} U_{2}^{*}\left|S_{2}^{*}\right|^{\frac{1}{2}}$. Let $\widetilde{S_{2}^{*}}=V_{2}^{*}\left|\widetilde{S}_{2}^{*}\right|$ be the polar decomposition of $\widetilde{S_{2}^{*}}$ and $\widehat{S_{2}^{*}}=\left|\widetilde{S}_{2}^{*}\right|^{\frac{1}{2}} V_{2}^{*}\left|\widetilde{S}_{2}^{*}\right|^{\frac{1}{2}}$. Applying $[6$, Corollary 1] to

$$
S_{1} X_{21}=X_{21} N_{2}
$$

and

$$
\left.S_{1} X_{22}\left|S_{2}^{*}\right|^{\frac{1}{2}}\left|{\widetilde{S_{2}}}_{2}^{* \frac{1}{2}}=X_{22}\right| S_{2}^{*}\right|^{\frac{1}{2}}\left|{\widetilde{S_{2}^{*}}}^{*}\right|^{\frac{1}{2}}\left(\widehat{S_{2}^{*}}\right)^{*}
$$

together with Theorem 8 to

$$
N_{1} X_{12}=X_{12} S_{2},
$$

we have $X_{21}=0, X_{22}=0$, and $X_{12}=0$. The rest of the proof is similar to the proof of Theorem 8 .

In Theorem 8 above if $X$ is a quasiaffinity then $\overline{R(X)}=\mathcal{H}_{1}$ and $N(X)^{\perp}=\mathcal{H}_{2}$. Hence $T_{1}$ and $T_{2}$ are unitarily equivalent normal operators. Thus we can obtain an improvement to Corollary 7 as follows.

COROLLARY 10. Let $T_{1} \in L\left(\mathcal{H}_{1}\right)$ be a log-hyponormal operator and let $T_{2} \in L\left(\mathcal{H}_{2}\right)$ be a normal operator. If there exists a quasiaffinity $X \in L\left(\mathcal{H}_{2}, \mathcal{H}_{1}\right)$ such that $T_{1} X=X T_{2}$, then $T_{1}$ and $T_{2}$ are unitarily equivalent normal operators.

Problem. Is it possible to replace the normality of $T_{2}$ in Corollary 10 with an isometry or a spectral operator?

\section{Log-hyponormal operators quasisimilar to isometries or spectral operators.}

THEOREM 11. Let $T_{1} \in L\left(\mathcal{H}_{1}\right)$ be log-hyponormal and let $T_{2} \in L\left(\mathcal{H}_{2}\right)$ be an isometry. If $T_{1}$ and $T_{2}$ is quasisimilar, then $T_{1}$ and $T_{2}$ are unitarily equivalent unitary operators.

Proof. There exist quasiaffinities $X$ and $Y$ such that $T_{1} X=X T_{2}$ and $Y T_{1}=T_{2} Y$. Since $T_{1}$ is invertible and $Y T_{1}=T_{2} Y, T_{2}$ has dense range. Hence $T_{2}$ is unitary. Thus $T_{1}$ and $T_{2}$ are unitarily equivalent unitary operators by Corollary 10 . 
Recall that a spectral operator (in the sense of Dunford) is an operator with a strongly countably additive resolution of the identity defined on the Borel sets of the complex plane. If $T$ is spectral, then it has the canonical decomposition $T=S+Q$, where $S$ and $Q$ are its scalar and its radical parts, respectively. For a thorough discussion of spectral operators see [7]. Hoover [12] studied quasisimilar spectral operators. It was proved that $M$-hyponormal spectral operators and $p$-hyponormal spectral operators are normal in [8] and [13], respectively. We conclude with the result that log-hyponormal spectral operators are normal.

THEOREM 12. Let $T_{1} \in L\left(\mathcal{H}_{1}\right)$ be a log-hyponormal operator and let $T_{2} \in L\left(\mathcal{H}_{2}\right)$ be a spectral operator. If there exists a quasiaffinity $X \in L\left(\mathcal{H}_{2}, \mathcal{H}_{1}\right)$ such that $T_{1} X=$ $X T_{2}$, then $T_{1}$ is normal, $T_{2}$ is a scalar operator, and $T_{2}$ is similar to $T_{1}$.

Proof. There exists a quasiaffinity $Y$ such that $\widetilde{T}_{1} Y=Y T_{1}$, by the same arguments as in the proof of Lemma 3 , and so we have that $\widetilde{T}_{1}(Y X)=(Y X) T_{2}$. Hence $Y X$ is also a quasiaffinity and so $\widetilde{T}_{1}$ is normal by $[\mathbf{8}$, Corollary 4$]$. If follows that $T_{1}\left(=\widetilde{T}_{1}\right)$ is normal by [16]. Thus $T_{2}$ is a scalar-type spectral operator and similar to $T_{1}$ by [9, Corollary 4].

COROLlary 13. If $T \in L(\mathcal{H})$ is a log-hyponormal spectral operator, then $T$ is normal.

\section{REFERENCES}

1. A. Aluthge, On $p$-hyponormal operators for $0<p<1$, Integral Equations Operator Theory 13 (1990), 307-315.

2. A. Aluthge and D. Wang, An operator inequality which implies paranormality, Math. Inequalities and Applications 2 (1999), 113-119. $169-178$.

3. T. Ando, Operators with a norm condition, Acta Sci. Math. (Szeged) 33 (1972), 689-702.

4. J. Conway, On quasisimilarity for subnormal operators, Illinois J. Math. 24 (1980),

5. B. P. Duggal, Quasi-similar p-hyponormal operators, Integral Equations Operator Theory 26 (1996), 338-345.

6. B. P. Duggal, On dominant operators, Arch. Math. (Basel) 46 (1986), 353-359.

7. N. Dunford and J. T. Schwartz, Linear operators, Part III: Spectral operators (Interscience, New York, 1971).

8. C. K. Fong, On $M$-hyponormal operators, Studia Math. 65 (1979), 1-5.

9. M. Fujii, C. Himeji and A. Matsumoto, Theorems of Ando and Saito for $p$-hyponormal operators, Math. Japonica 39 (1994), 595-598.

10. F. Hansen, An inequality, Math. Ann. 246 (1980) 249-250.

11. E. Heinz, Beiträge zur Störungstheorie der Spektralzerlegung, Math. Ann. 123 (1951), $415-438$.

12. T. B. Hoover, Quasisimilarity of operators, Illinois J. Math. 16 (1972), 678-686.

13. I. H. Jeon and B. P. Duggal $P$-hyponormal operators and quasisimilarity, Integral Equations Operator Theory, to appear.

14. K. Löwner, Über monotone Matrixfunktionen, Math. Z. 38 (1934), 177-216.

15. J. G. Stampfli and B. L. Wadhwa, An asymmetric Putnam-Fuglede theorem for dominant operators, Indiana Univ. Math. J. 25 (1976), 359-365.

16. K. Tanahashi, On log-hyponormal operators, Integral Equations Operator Theory 34 (1999), 364-372.

17. K. Tanahashi, Putnam's inequality for log-hyponormal operators, Integral Equations Operator Theory, to appear. 
18. A. Uchiyama and K. Tanahashi, Fuglede-Putnam's theorem for $p$-hyponormal or loghyponormal operators, Glasgow Math. J. 44 (2002), 397-410.

19. L. R. Williams, Quasisimilarity and hyponormal operators, J. Operator Theory 5 (1981), $127-139$

20. D. Xia, Spectral theory of hyponormal operators (Birkhäuser, Basel, 1983).

21. T. Yoshino, The p-hyponormality of the Aluthge transform, Interdisciplinary Information Sciences 3 (1997), 91-93. 